\title{
Playing games with numbers
}

SIR - Peter Stanbury( Nature 7 July, p.11) presented an interesting account of the ubiquity of $\pi$ in particle physics. Your accompanying comment led me to do some quick number crunching of my own. I soon discovered that, if the number of the Nature volume containing the article (304) is divided into the issue number (5291) and the resulting quotient is divided by the page number on which the article is printed (11), the resulting number, 1.771 , is 99.9 per cent of the value $\pi^{1 / 2}$. Surely this is simply a coincidence, or is it?

\section{Department of Ophthalmology,}

R.B. ROSENBERG University of Louisville, Louisville, Kentucky 40202, USA

SIR - In "The temptations of numerology" (Nature 7 July, p.11) you ask “. . . why are the relationships never exact?" The answer is that sometimes they are and you said yourself that "the numerologists do have a few successes to their credit". Some famous examples, which perhaps led to modern physics and society, were:

(1) Kirkhoff's observation in 1857 that the ratio of the electrical units was equal to the velocity of light. It was explained by Riemann in 1858 (see Max Mason and Warren Weaver, The Electromagnetic Field (1929), p.x).

(2) In 1885 Balmer gave a formula that fitted the spectral lines of hydrogen. It was explained in 1913 by Niels Bohr, and better by Dirac and Pauli in 1926.

(3) Kepler's third law was numerological, and was explained by Newton.

All three of these examples are of great historical and scientific interest. They were fairly accurate and also simple, and were right. Eddington's attempts, for elementary particles, were accurate at the time, forced, and wrong. There is of course such a thing as obviously bad numerology. The worst published one that I can recall appeared, believe it or not, in Nature! (185, $602 ; 1960$ )

I.J. GOOD

\section{Department of Statistics, \\ Virginia Polytechnic Institute and State University, Blacksburg, Virginia 24061, USA}

SIR - Your correspondent Peter Stanbury (Nature 7 July, p.11) has applied considerable effort and ingenuity to numerical speculations about mass ratios of fundamental particles. However, like many amateurs in science, he has missed one important point: the significance of (in)accuracies. Specifically, he takes the sum of the masses of the lightest baryon octet. He then finds that the ratios of these mass sums to the proton mass are 3.14006 and 9.812 respectively, and suggests that these numbers are "very nearly $\pi$ or 3.14159 " and "close to $\pi^{2}$ or 9.86960 ".

Now, using the values given in the 1982 tables $^{1}$, I obtain $3.13935 \pm 0.00066$ and $9.81460 \pm 0.00062$ for these ratios; these are not as quoted by Mr Stanbury, presumably because he used older tables. However, the chief significance lies in the errors (standard deviations here). They exclude Mr Stanbury's assignments by 3.4 standard deviations for the first and no less than 89 for the second.

In general (and it is sad to have to state this in Nature) any experimental result has at least two parts: the measurement itself, and the error on it. If any theory purports to predict the result, we have an immediate indication of the worth of that theory. On this basis Mr Stanbury's theory is in error by some 90 standard deviations; it therefore fails.

To forestall one possible defence, I accept that Mr Stanbury's later relations (2) and (3) do pass this particular test; but two "hits" and two bad "misses" do not justify any theory. (Since his other results depend on the choice of mass units, I decline to discuss them here.) To forestall another, I am a professional physicist, and therefore perhaps one of that stuffy establishment so feared by Mr Stanbury. But surely it does not take years of training and experience to realize that (to illustrate) if a theory predicts $x$, and if experiment says $2 x \pm 0.1 x$, then that theory is wrong.

\section{Schweizerisches Institut für \\ Nuklearforschung.} JOHN F. CRAWFORD 5234 Villigen, Switzerland

1. Phys.Lett. 111B, April 1982.

\section{Sizewell agony}

SIR - The opinion page article "End the Sizewell agony" (Nature 4 August, p.382) contains many factual errors. The most notable of these is the one that serves as its premise. In asserting that the Sizewell inquiry is causing a delay to the construction programme of this power station, it confuses the inquiry with a proper investigation of the economics, safety and other aspects of the proposal of the Central Electricity Generating Board (CEGB).

Despite ministerial assurances to the contrary, the inquiry is taking place in advance of the CEGB having prepared a design for construction. The Nuclear Installations Inspectorate (NII) will not grant a licence for construction until 80 outstanding safety issues are resolved. This requirement, they have repeatedly maintained, is entirely independent of the inquiry, and is unlikely to be satisfied until the end of 1984 at the earliest - long after the inquiry is over. The delay in the construction programme is due solely to the CEGB's difficulty in designing a reactor which conforms to British safety standards while also meeting its cost predictions. The first design for this reactor was rejected in 1981 (after 31/2 years of design effort) by the $C E G B$ because it was too expensive. Far from idly kicking their heels, the design teams are frantically trying to make up for lost time.

The inquiry is an absurdity for precisely the opposite reason to the one you advance. It cannot deal with many issues which are crucial to the cost and safety of the reactor until a design is produced which the CEGB intends to construct, and which has been passed by the NII. Meanwhile, electricity consumers, not taxpayers, are footing the bill - the same consumers who, according to the CEGB's own figures, have underwritten more than 25 years of uneconomic nuclear power. Despite the apparent opportunity the inquiry provides fully to investigate these questions, you may rest assured that the public is likely to have little influence on whether, or indeed when, the CEGB's programme will go ahead.

Friends of the Earth Ltd,

RENEe Chudleigh WILlIAM CANNELL

377 City Road London ECIV INA, UK

\section{Life's thermal history}

SIR - Presumably the organisms living around abyssal thermal vents get most of their energy by catalysing reactions between reducing substances coming up the vent, and oxygen spreading down from the distant atmosphere. Baross and Deming ${ }^{1}$ do not explicitly say whether oxygen was present in the chambers in which they grew bacteria at unusually high temperatures. If it was present, their results may have little bearing on the origins, as opposed to the potentialities, of life.

The observation is extremely interesting. But it is not as "astounding" as Walsby" suggests. There is a good deal of parochial thinking in his annotation. Our mode of living is no doubt excellent in our environment. Hot springs and similar sites on Earth's surface are too evanescent, on an evolutionary time scale, to encourage extensive biological exploitation. However, as I have often pointed out (see, for example, ref.3), if an environment exists for a few million years where there is an exploitable chemical disequilibrium, it is unreasonable to assume that organisms will not adapt so as to make use of it. The essential points are duration and disequilibrium. It therefore seems unlikely that organisms flourish further down the hydrothermal vent because it is difficult to envisage a reaction which they could use there which would not already have taken place without biological aid.

N.W. PIRIE

\section{Harpenden, Herts, UK}

Baross, J.A. \& Deming, J.W. Nature 303, 423-426 (1983). Walsby, A.E. Nature 303, 381 (1983).

Pirie, N.W. Nature 235, 287 (1972); 296, 796 (1982). 